\title{
Assessment of Malaria Microscopic Diagnosis Performance of Laboratory Professionals in Addis Ababa's Public Health Facilities
}

\author{
Leykun Demeke Gebrekidan ${ }^{1, ~}$, Honelgn Nahusenay Hiruy ${ }^{2}$ \\ ${ }^{1} \mathrm{ICAP}$ at Columbia University, Addis Ababa, Ethiopia \\ ${ }^{2}$ Addis Continental Institute of Public Health, Addis Ababa, Ethiopia \\ Email address: \\ labkey2004@yahoo.com (L. D. Gebrekidan),h.nahu2000@gmail.com (H. N. Hiruy) \\ ${ }^{*}$ Corresponding author
}

\section{To cite this article:}

Leykun Demeke Gebrekidan, Honelgn Nahusenay Hiruy. Assessment of Malaria Microscopic Diagnosis Performance of Laboratory Professionals in Addis Ababa's Public Health Facilities. Biomedical Sciences. Vol. 5, No. 1, 2019, pp. 1-6. doi: 10.11648/j.bs.20190501.11

Received: November 28, 2018; Accepted: January 5, 2018; Published: January 31, 2019

\begin{abstract}
Cross-sectional study was conducted using panel blood films and questioner to assess detection \& identification performance of laboratory professionals' and identify factors affecting the performance of malaria microscopic diagnosis. Study participants had $91.7 \%$ (95\% CI: 89.96 - 93.44) agreement for detection of malaria parasites, 67.63\% (95\% CI: 64.91 70.35) species identification agreement for Plasmodium falciparum, $5.08 \%$ false positive and $21.04 \%$ false negative results. Correct species identification percentage for Plasmodium falciparum were 60.9\% (510), Plasmodium vivax 59.17\% (371) and Mixed (Plasmodium falciparum and Plasmodium vivax) 25\% (53) were also identified in the study. In addition, sensitivity 94.69\% (95\% CI: $93.02-96.36)$ and specificity of 79.71 (95\% CI: $75.22-84.2)$ were calculated from panel blood film results. The most frequent type of misdiagnosis was 85(40.09\%) mixed BFs diagnosed as Plasmodium vivax, 67 (31.6\%) mixed BFs as Plasmodium falciparum and 218(26\%) Plasmodium falciparum BFs as Plasmodium vivax. Moreover, only 18(8.5\%) laboratory professionals were participated in external quality assessment. From multiple logistic regression analysis training was the major factor for species identification percent agreement performance improvement of laboratory professionals. It showed statistical significance with p-value $<0.05$ and untrained laboratory professionals were $64 \%$ less likely to perform $\geqslant$ $85 \%$ agreement of species identification. Training of laboratory professionals on malaria microscopic diagnosis help to improve the accuracy and reliability of reported results. This will help to provide the right and recommended medication and patient management.
\end{abstract}

Keywords: Malaria, Microscopy, Diagnosis, Performance, Laboratory Professionals, Addis Ababa \& Public Health Facilities

\section{Introduction}

Microscopy of giemsa stained both thick and thin blood film (BF) is the gold standard and preferred option for diagnosis of malaria [1]. Parasitological testing is the only way to diagnose malaria accurately in febrile patients. If malaria is suspected on clinical grounds, it is mandatory to obtain the laboratory confirmation for the presence or absence of malaria parasites. According to 2011-2015 national strategic plan $100 \%$ of suspected malaria cases are expected to be diagnosed using microscopy or rapid diagnostic test (RDT) within 24 hours of fever onset. Proper diagnosis provides accurate and reliable result and is used for optimal treatment and save lives [1, 2, 3].

Malaria microscopic diagnosis (MMD) is better than clinical diagnosis for the proper management of malaria cases and other febrile illnesses. Misdiagnosis is the major challenge for the diagnosis of malaria using microscopy. This is due to the competency ability of laboratory professionals and lack of adherence to malaria microscopy standards [3]. Malaria treatment based on the laboratory diagnosis is the most preferred option and has its own advantage than the presumptive diagnosis. Misdiagnosis of malaria infection facilitates further transmission of malaria to the community 
and cause misuse of anti-malaria drugs. Moreover, unable to identify patients not having malaria infection will lead to the exclusion of other options for febrile illness diagnosis and treatment. Since early 2010, World Health Organization(WHO) has recommended prompt parasitological confirmation by microscopy or RDT for all patients suspected of having malaria infection, before treatment is started $[4,5]$.

MMD performance depend on the competency of laboratory professionals, quality of reagents, training status on MMD, adherence to the standards, participation to external quality assessment(EQA) and microscope functionality. Panel testing or proficiency test is one type of EQA method. It is used to assess the performance of laboratory professionals on malaria parasite detection and identification using prepared known panel BFs. Panel BFs is prepared in a set of containing both positive and negative BFs according to the standards. Panel BFs species is detected and identified by qualified skilled laboratory professionals [3, 5].

Addis Ababa is located in a highland which is assumed to be malaria free. However, the city hosts peoples from different parts of the country and abroad. Currently the movement of people to and fro the city increases due to different reasons. In addition, the residents of the town have a chance of going to the malarious area and also peoples from the malarious area coming to the city.

Laboratory professionals working in Addis Ababa's health facilities are not exposed to positive malaria parasite $\mathrm{BF}$ readings frequently. So their malaria parasite detection and identification skill my decline over time, thus assessing their competency skill is a key point to identify major gaps affecting their performance. Clinicians in malarious areas may treat false negative cases. But since Addis Ababa is malaria free area the chance of false negative cases to be treated is low. The residents of the city are less immune to plasmodium species and if a patient is misdiagnosed as negative then its effect will be dangerous. For these reasons efficient MMD service is required to provide accurate and reliable laboratory result to febrile patient(s).

\section{Methods}

\subsection{Study Area and Population}

Addis Ababa is the capital and largest city of Ethiopia. The city divided in to ten sub cities and lies at an altitude of 2,300 meters above sea level. During the study period 67 Health Centers and 6 Hospitals owned by Addis Ababa regional health bureau giving malaria microscopy diagnosis service. Two hundred eleven laboratory professionals were participated during the data collection period, November 2013 to February 2014, from 47 Health Centers and 3 Hospitals.

\subsection{Study Design}

Cross sectional study design was conducted in public health facilities of Addis Ababa.

\subsection{Data Collection}

Laboratory professionals at the study health facilities were provided set of panel blood films. Each BF had both thick and thin BF on the same slide, the thin BF fixed with absolute methanol and stained with giemsa stain working solution. The panel BFs were prepared, fixed, stained and mounted according to WHO BF preparation standards. Study participant laboratory professionals were examined BFs using microscope found in their health facilities and reported the finding on provided reporting format. The report of panel BFs results were included the type of malaria parasite diagnosed (species) and stage for those positive BFs. We were used set of panel BFs for each laboratory professionals. Each set of panel BFs were consisted of four Plasmodium falciparum, three Plasmodium vivax and one Mixed (Plasmodium falciparum and Plasmodium vivax) infections with parasite densities ranging from 5440 - 23,160 parasites $/ \mu 1$ and two negative BFs. A total of 211 laboratory professionals were examined 2096 BFs; 838(40\%) were Plasmodium falciparum, 627(30\%) Plasmodium vivax, 212 (10\%) Mixed (Plasmodium Falciparum and Plasmodium vivax) and the rest $419(20 \%)$ negative BFs. After completion of malaria microscopy diagnosis, interview based on prepared study questioner were filled and the finding recorded accordingly. Observations were also one way of data obtaining procedure during questioner assessment of the laboratory professionals.

\section{Results}

\subsection{Characteristics of Laboratory Professionals Participated in the Study}

A total of 211 laboratory professionals were participated in the study: 98(46.44\%) female, 130(61.61\%) diploma, $81(38.39 \%)$ BSc and above and $43(20.38 \%)$ trained on MLD (See Table 1).

Table 1. Characteristics of laboratory professionals participated in the study.

\begin{tabular}{llll}
\hline Factors & & Frequency & Percent \\
\hline \multirow{2}{*}{ Sex } & Female & 98 & $46.44 \%$ \\
& Male & 113 & $53.56 \%$ \\
Age & $20-27$ & 139 & $65.88 \%$ \\
& $28-54$ & 72 & $34.12 \%$ \\
Service Year & $<5$ Years & 118 & $55.92 \%$ \\
Training on MLD & $\geq 5$ Years & 93 & $44.08 \%$ \\
\hline
\end{tabular}




\begin{tabular}{llll}
\hline Factors & & Frequency & Percent \\
\hline \multirow{4}{*}{ Education Level } & Yes & 43 & $20.38 \%$ \\
& Diploma & 130 & $61.61 \%$ \\
Marital Status & BSc and above & 81 & $38.39 \%$ \\
& Never Married & 155 & $73.46 \%$ \\
SOPs & Ever Married & 56 & $26.54 \%$ \\
& Used & 42 & $19.9 \%$ \\
National EQA Guidelines & Not Used & 127 & $60.2 \%$ \\
Job Aid & Not Available & 42 & $19.9 \%$ \\
& Not Available & 211 & $100 \%$ \\
Type of Blood film Prepared & Not Available & 211 & $100 \%$ \\
& Only Thin Film & 67 & $31.8 \%$ \\
IQC for Giemsa stain & Only Thick film & 73 & $34.6 \%$ \\
& Both thin and thick on the same slide & 71 & $33.6 \%$ \\
EQA Participation & No & 126 & $59.7 \%$ \\
& Yes & 85 & $40.3 \%$ \\
\hline
\end{tabular}

\subsection{Performance of Laboratory Professionals}

Among the total examined BFs 85 false positive, 89 false negative, 1588 true positive, 334 true negative and 654 species misdiagnosis were detected and identified. Study participant laboratory professionals were scored $91.7 \%$ (95\% CI: 89.96 - 93.44) agreement for detection of malaria parasite, $67.63 \% \quad(95 \% \quad C I: 64.91$ - 70.35$)$ species identification agreement for Plasmodium falciparum, 5.08\% false positive and $21.04 \%$ false negative results. Moreover, their sensitivity and specificity were $94.69 \%$ (95\% CI: 93.02 - 96.36) and 79.71\% (95\% CI: 75.22-84.2) respectively.

\subsection{Species Identification Performance of Laboratory Professionals}

According to national treatment guidelines species identification is the key for accurate treatment provision for malaria infected patients. Generally, study participant laboratory professionals in Addis Ababa were identified correctly malaria species in $60.5 \%$ (1268) $(95 \%$ CI: 60.41$60.59)$ BFs. Correct species identification for mixed BFs were $25 \%$ (53) (95\% CI: $24.94-25.06)$ and it was a very minimum diagnostic result compared to other species (See Table 2).

Table 2. Correct species identification performance of laboratory professionals in Addis Ababa.

\begin{tabular}{llll}
\hline Species & Total BF Examined & Correctly Identified & Percent correctly Identified (95\% CI) \\
\hline Plasmodium Falciparum & 838 & 510 & $60.9 \%(60.76-60.96)$ \\
Plasmodium Vivax & 627 & 371 & $59.17 \%(59.09-59.25)$ \\
Mixed (Plasmodium Falciparum \& Plasmodium Vivax) & 212 & 53 & $25 \%(24.94-25.06)$ \\
Negative & 419 & 334 & $79.71 \%(79.65-79.77)$ \\
Total & 2096 & 1268 & $60.5 \%(60.41-60.59)$ \\
\hline
\end{tabular}

\subsection{Misdiagnosis}

Misdiagnosis is an error encountered by the laboratory professionals during MMD. There were three types of misdiagnosis; species misdiagnosis, false negative and false positive. In the study $39.5 \%(828)$ misdiagnosis were reported. The most frequent type of misdiagnosis was $40.09 \%$ (85) mixed species reported as Plasmodium vivax and $26 \%$ (218) Plasmodium falciparum as Plasmodium vivax (See Table 3).

Table 3. Misdiagnosis type and performance of laboratory professionals in Addis Ababa.

\begin{tabular}{llll}
\hline Misdiagnosis Type & & Frequency & Percent Misdiagnosis \\
\hline & Pf as Pv & 218 & $26 \%$ \\
& Pf as Mixed & 73 & $8.7 \%$ \\
Species Misdiagnosis & Pv as Pf & 159 & $25.36 \%$ \\
& Pv as Mixed & 52 & $8.29 \%$ \\
& Mixed as Pf & 67 & $31.6 \%$ \\
& Mixed as Pv & 85 & $40.09 \%$ \\
& Pf as Neg & 37 & $4.4 \%$ \\
False Negative & Pv as Neg & 45 & $7.18 \%$ \\
& Mixed as Neg & 7 & $3.3 \%$ \\
False Positive & Neg as Pf & 36 & $8.59 \%$ \\
& Neg as Pv & 49 & $11.7 \%$ \\
& Neg as Mixed & 0 & $0 \%$ \\
& Total & 828 & $39.5 \%$ \\
\hline
\end{tabular}

Note: Pf - Palasmodium falciparum, $\mathrm{Pv}$ - Plasmodium vivax, Mixed - Pf \& $P v$ and $\mathrm{Neg}$ - Negative for malaria parasite. 


\subsection{Sensitivity and Specificity}

Sensitivity and specificity tells the ability of laboratory professionals to detect malaria parasites from positive blood films and absence of malaria parasite from negative blood films respectively. Trained laboratory professionals had better performance of sensitivity and specificity $97.38(95 \%$ CI: $94.49-100)$ and 90.79 (95\% CI: 83.97 - 97.43) respectively (See Table 4).

Table 4. Sensitivity and specificity performance of laboratory professionals in Addis Ababa.

\begin{tabular}{llllll}
\hline Category & & Number of Lab Professionals & Total BF Examined & Sensitivity (95\% CI) & Specificity (95\% CI) \\
\hline \multirow{2}{*}{ Training Status } & Trained & 43 & 430 & $97.38(94.49-100)$ & $90.79(83.97-97.43)$ \\
& Untrained & 168 & 1666 & $94(92.05-95.95)$ & $76.88(71.59-82.17)$ \\
\multirow{2}{*}{ Education Level } & Bsc & 80 & 791 & $97(95.19-98.81)$ & $86.71(80.37-93.03)$ \\
& Diploma & 131 & 1305 & $93.3(72.88-77.72)$ & $75.48(69.44-81.52)$ \\
\multirow{2}{*}{ Service Year } & $\geq 5$ Years & 94 & 931 & $95.84(93.7-97.98)$ & $84.95(78.98-90.92)$ \\
& $<5$ Years & 117 & 1165 & $93.78(91.32-96.24)$ & $75.54(69.07-82.01)$ \\
\hline
\end{tabular}

\subsection{Factors Affecting Performance of Malaria Microscopy Diagnosis}

Binary logistic regressions analysis was done by taking percent agreement as dependent variable (outcome) and sex, age, service year, training on malaria laboratory diagnosis, educational level and marital status taken as independent variables. Factors which show statistical significance by binary logistic regression were training on malaria laboratory diagnosis and educational level with $\mathrm{P}$-value $<0.05$. In the multiple logistic regression analysis training was the only factor which shows statistical significance with $p$ - value of 0.01 . Trained laboratory professionals had better performance than untrained laboratory professionals. Untrained laboratory professionals were $64 \%$ less likely to perform $\geq 85 \%$ species identification agreement for Plasmodium falciparum (AOR 0.36 (95\% CI: 0.16-0.78)) (See Table 5).

Table 5. Multiple logistic regression analysis for factors affecting species identification percent agreement performance of Laboratory Professionals.

\begin{tabular}{|c|c|c|c|c|c|}
\hline \multirow{2}{*}{ Factors } & \multicolumn{2}{|c|}{ Species Identification Percent Agreement for Pf } & \multirow{2}{*}{ COR $(95 \% \mathrm{CI})$} & \multirow{2}{*}{$A O R * *(95 \% C I)$} & \multirow{2}{*}{ P-value* } \\
\hline & $<85 \%$ & $\geq 85 \%$ & & & \\
\hline \multicolumn{6}{|l|}{ Sex } \\
\hline Female & 75 & 23 & $0.89(0.47-1.67)$ & $0.90(0.46-1.78)$ & 0.77 \\
\hline Male & 84 & 29 & 1 & 1 & \\
\hline \multicolumn{6}{|l|}{ Age } \\
\hline $20-27$ & 105 & 34 & $0.97(0.50-1.88)$ & $1.53(0.68-3.46)$ & 0.31 \\
\hline $28-54$ & 54 & 18 & 1 & 1 & \\
\hline \multicolumn{6}{|l|}{ Service Year } \\
\hline$<5$ years & 94 & 24 & $0.59(0.32-1.11)$ & $0.73(0.33-1.60)$ & 0.43 \\
\hline$\geq 5$ years & 65 & 28 & 1 & 1 & \\
\hline \multicolumn{6}{|c|}{ Training on MLD } \\
\hline No & 135 & 33 & $0.31(0.15-0.63)$ & $0.36(0.16-0.78)$ & 0.01 \\
\hline Yes & 24 & 19 & 1 & 1 & \\
\hline \multicolumn{6}{|c|}{ Educational Level } \\
\hline Diploma & 105 & 25 & $0.48(0.25-0.90)$ & $0.66(0.33-1.34)$ & 0.25 \\
\hline BSc and Above & 54 & 27 & 1 & 1 & \\
\hline
\end{tabular}

Key: * Significance for $\mathrm{p} \leq 0.05 * *$ Adjusted for Sex, Age, Service year, Training on MLD and Educational Level.

\section{Discussion}

The study identified that, MMD performance of laboratory professionals in Addis Ababa were 91.7\% (95\% CI: $89.96-$ 93.44) agreement for detection of malaria parasite and $67.63 \%$ (95\% CI: $64.91-70.35)$ species identification agreement for Plasmodium falciparum. In addition, 67 (31.6\%) mixed BFs diagnosed as Plasmodium falciparum, 218(26\%) Plasmodium falciparum BFs as Plasmodium vivax and $11.7 \%$ (49) negative BFs were diagnosed as Plasmodium vivax. Detection was higher (percent agreement - 91.7\%) than identification (species identification percent agreement for Plasmodium falciparum- 67.63\%). So the study identified that there was higher performance problem on species identification.
Misdiagnosis results cause provision of incorrect antimalaria medication for the patient due to species misdiagnosis and false positive results. Moreover, false negative results like Plasmodium falciparum were reported as negative in $4.4 \%$ (37) and mixed as negative in $3.33 \%$ (7) BFs cause malaria infected patients might not getting proper medication, worsen the disease condition of the patient and facilitate further transmission of malaria parasite in the community. Similar condition indicated from the study conducted in Uganda on malaria laboratory diagnosis and treatment $[12,13]$.

Misdiagnosis were higher among non-trained than trained laboratory professionals. From the study conducted on misclassification of plasmodium infection by microscopy, post training performance of malaria parasite species 
identification was increased compared to pre-training. This showed that training brought a remarkable change in species identification [15]. Trained laboratory professionals' performance was better than non-trained laboratory professionals on MMD. This was also seen in other studies on MMD performance assessment [6, 7, 8, 9, 10, 11]. From the study conducted in Kenya misdiagnosis were reduced by providing training, sensitivity improved by a mean of $14 \%$ (CI 9-19\%) from 77\% baseline (CI 73-81\%), while specificity improved by a mean of $17 \%$ (CI $11-23 \%$ ) from $76 \%$ (CI 70-82\%) baseline [8]. In addition, from the study conducted in Uganda, after training sensitivity improved from $84 \%$ to $95 \%$ and specificity improved from $87 \%$ to $97 \%$ $[14,15]$. Similarly, the study identified that trained laboratory professionals had higher sensitivity and specificity $(97.38 \%$ $\& 94 \%)$ than untrained laboratory professionals $(90.7 \%$ \& $76.88 \%$ ) respectively.

The quality of BF preparation was one factor for the performance improvement of MMD [14, 15]. The recommended $\mathrm{BF}$ preparation for diagnosis of malaria parasite is doing both thin and thick BFs on the same slide using $2 \mu 1$ and $6 \mu 1$ whole blood respectively. The study was identified $31.8 \%$ (67) laboratory professionals were prepared only thin BF, $34.6 \%$ (74) only thick BF and 33.6\% (71) thick and thin BF on the same slide. Moreover, BFs performed by $89.1 \%$ (1880) laboratory professionals were not met the quality of good BF for MMD.

Internal quality control, used to check the quality of giemsa stains, was performed by $40.3 \%$ (85) laboratory professionals. In addition, only $8.5 \%$ (18) laboratory professionals were participated in EQA, which is a very minimal number. EQA for malaria microscopy diagnosis used to identify deficiencies and take corrective action. It is the key for the performance improvement of the accuracy and reliability of reported results in MMD. Moreover, EQA should be supported by the availability and usage of quality reagents and documents like standard operating procedures, guidelines and job aids $[16,17,18,19,20]$.

\section{Conclusions and Recommendation}

Misdiagnosis is the key cause for misusage of anti-malaria medications, to become worse the disease condition of malaria infected patients and allow further transmission of the parasite in the community. Misdiagnosis of malaria parasites was higher among untrained than trained laboratory professionals. Training on MMD and adherence to the standards improve performance of laboratory professionals in order to obtain accurate and reliable results. This will bring remarkable change to provide the right and recommended anti-malaria medication for better management of malaria parasite infected patients, minimize further transmission of the plasmodium species in the community and identify patients not infected with malaria parasites in order to consider other febrile illnesses differential diagnosis and treatment.

\section{Abbreviations}

\author{
BF - Blood Film \\ EQA - External Quality Assessment \\ MLD - Malaria Laboratory Diagnosis \\ MMD - Malaria Microscopic Diagnosis \\ RDT - Rapid Diagnostic Test \\ WHO - World Health Organization
}

\section{Authors Contributions}

LD being the principal investigator, developed the study protocol \& tools, done the data management and wrote the first draft manuscript. HN assisted with the study design and protocol development, and helped to draft the manuscript. LD and HN contributed to data analysis and interpretation. Both authors read and approved the final manuscript.

\section{Acknowledgements}

The authors would like to express appreciation to Tewabech Lemma, Sintayehu Gebresilassie and Gemechu Tadesse for their kind cooperation for the detection and identification of panel blood films. We also acknowledge University of Gondar, Addis Continental Institute of Public Health and Addis Ababa Regional Health Bureau for the permission to conduct the research. Finally, the authors would like to acknowledge Kidist Mikre for the data collection and study participant laboratory professionals from selected public health facilities in Addis Ababa.

\section{Ethics Approval and Consent to Participate}

The study had ethical approval from University of Gondar and Addis Continental Institute of Public Health ethical review board. Permission were asked and permitted from Addis Ababa Regional Health Bureau in order to conduct the study based on the ethical review board approval. In addition, written consent was obtained from all study participant laboratory professionals.

\section{Consent for Publication}

Not applicable.

\section{Availability of Data and Material}

All data and materials used for the study available and kept in safe place confidentially.

\section{Competing Interests}

The authors declare that they have no competing interests 


\section{References}

[1] World Health Organization: A practical handbook management of severe malaria, Third Edition 2011.

[2] EHNRI: Manual for the Laboratory Diagnosis of Malaria first edition - September, 2012

[3] EHNRI: Malaria Laboratory Diagnosis External Quality Assessment Scheme Guidelines, September, 2009.

[4] World Health Organization: Universal access to malaria diagnostic testing an operation manual, 2011.

[5] WHO: Malaria Microscopy Quality Assurance Manual, Version 1. March 2009.

[6] John Frean: Microscopic determination of malaria parasite load: role of image analysis. Microscopy: Science, Technology, Applications and Education A. Méndez-Vilas and J. Díaz (Eds.) 2010, 862-866.

[7] Alexander N, Schellenberg D, Ngasala B, Petzold M, Drakeley C, Sutherland C: Assessing agreement between malaria slide density readings. Malaria Journal 2010, 9:4.

[8] Ohrt C, Obare P, Nanakorn A, Adhiambo C, Awuondo K, Prudhomme W, Remich S, Martin K: Establishing a malaria diagnostics centre of excellence in Kisumu, Kenya. Malaria Journal 2007, 6:79.

[9] O'Meara W, Barcus M, Wongsrichanalai C, Muth S, Maguire J, Jordan R, Prescott W and McKenzie E: Reader technique as a source of variability in determining malaria parasite density by microscopy.Malaria Journal 2006, 5:118.

[10] Kyabayinze DJ, Achan J, Nakanjako D, Mpeka B, Mawejje H, Mugizi R, Kalyango JN, D’Alessandro U, Talisuna A, JeanPierre VG: Parasite-based malaria diagnosis: Are Health Systems in Uganda equipped enough to implement the policy? BMC Public Health 2012, 12:695.

[11] Namagembe A, Ssekabira U, Weaver M, Blum N, Burnett S, Dorsey G, Sebuyira LM, Ojaku A, Schneider G, Willis K and Yeka A: Improved clinical and laboratory skills after team based, malaria case management training of health care professionals in Uganda. Malaria Journal 2012, 11:44.

[12] Nankabirwa J, Zurovac D, Njogu JN, Rwakimari JB, Counihan H, Snow RW and Tibenderana JK: Malaria misdiagnosis in Uganda - implications for policy change. Malaria Journal 2009, 8:66.

[13] Zurovac D, Midia B, Ochola SA, English M, Snow RW: Microscopy and outpatient malaria case management among older children and adults in Kenya. Trop Med Int Health 2006, $11: 432-440$

[14] Kiggundu M, Nsobya SL, Kamya MR , Filler S, Nasr S, Dorsey $\mathrm{G}$, and Yeka A: Evaluation of a Comprehensive Refresher Training Program in Malaria Microscopy Covering Four Districts of Uganda. American Journal of Tropical Medcine and Hygine 2011, 84(5): 820-824.

[15] Obare P, Ogutu B, Adams M, Odera JS, Lilley K, Dosoo D, Adhiambo C, Owusu-Agyei S, Binka F, Wanja E and Johnson J: Misclassification of Plasmodium infections by conventional microscopy and the impact of remedial training on the proficiency of laboratory technicians in species identification. Malaria Journal 2013, 12:113.

[16] Mukadi P, Gillet P, Lukuka A, Atua B, Sheshe N, Kanza A, Mayunda JB, Mongita B, Senga R, Ngoyi J, Muyembe J, Jacobsb J \& Lejonh V.: External quality assessment of Giemsa-stained BF microscopy for the diagnosis of malaria and sleeping sickness in the Democratic Republic of the Congo. Bull World Health Organ 2013;91:441-448.

[17] Khan MA, Walley JD, Munir MA, Khan MA, Khokar NG, Tahir Z, Nazir A, Shams N: District level external quality assurance (EQA) of malaria microscopy in Pakistan: pilot implementation and feasibility. Malaria Journal 2011, 10:45.

[18] Mukadi P, Gillet P, Lukuka A, Atua B, Kahodi S, Lokombe J, Jean-Jacques Muyembe and Jacobs J: External quality assessment of malaria microscopy in the Democratic Republic of the Congo. Malaria Journal 2011, 10:308.

[19] Ashraf S, Kao A, Hugo C, Christophel EM, Fatunmbi B, Luchavez J, Lilley K and Bell D: Developing standards for malaria microscopy: external competency assessment for malaria microscopists in the Asia-Pacific. Malaria Journal 2012, 11:352.

[20] Frean J, Perovic O, Fensham V, McCarthy K, Gottberg A, Gouveia L, Poonsamy B, Dini L, Rossouw J., Keddy K, Alemu W, Yahaya A, Pierson A, Dolmazon V, Cognatc S \&. Ndihokubwayo JB: External quality assessment of national public health laboratories in Africa, 2002-2009. Bull World Health Organ 2012;90:191-199A. 